\title{
Stellar density profile and mass of the Milky Way bulge from VVV data ${ }^{\star}$
}

\author{
E. Valenti ${ }^{1}$, M. Zoccali ${ }^{2,3}$, O. A. Gonzalez ${ }^{4,5}$, D. Minniti ${ }^{3,6,7}$, J. Alonso-García ${ }^{8,3}$, E. Marchetti ${ }^{1}$, M. Hempel $^{2}$, \\ A. Renzini ${ }^{9}$, and M. Rejkuba ${ }^{1,10}$
}

1 European Southern Observatory, Karl Schwarzschild-Straße 2, 85748 Garching bei München, Germany e-mail: evalenti@eso.org

2 Instituto de Astrofísica, Pontificia Universidad Católica de Chile, Av. Vicuña Mackenna 4860, Santiago, Chile

3 Millennium Institute of Astrophysics, Av. Vicuña Mackenna 4860, 782-0436 Macul, Santiago, Chile

${ }^{4}$ European Southern Observatory, A. de Cordova 3107, Casilla 19001, Santiago 19, Chile

5 Institute for Astronomy, University of Edinburgh, Royal Observatory, Edinburgh EH9 3HJ, UK

6 Departamento de Ciencias Fisícas, Universidad Andrés Bello, República 220, Santiago, Chile

7 Vatican Observatory, V00120 Vatican City State, Italy

8 Unidad de Astronomía, Fac. de Ciencias Básicas, Universidad de Antofagasta, Antofagasta, Chile

9 Istituto Nazionale di Astrofisica, Osservatorio Astronomico di Padova, 35122 Padova, Italy

10 Excellence Cluster Universe, Boltzmannstr. 2, 85748 Garching, Germany

Received 4 October 2015 / Accepted 23 October 2015

\section{ABSTRACT}

\begin{abstract}
We present the first stellar density profile of the Milky Way bulge that reaches latitude $b=0^{\circ}$. The profile was derived by counting red clump stars within the colour-magnitude diagram that was constructed using the new PSF-fitting photometry from VISTA Variables in the Vía Láctea (VVV) survey data. The new stellar density map covers the area between $|l| \leq 10^{\circ}$ and $|b| \leq 4.5^{\circ}$ with unprecedented accuracy, allowing the stellar kinematics from the Giraffe Inner Bulge Spectroscopic Survey (GIBS) to be linked to the stellar mass density distribution. In particular, the location of the central velocity-dispersion peak from GIBS matches a high over-density in the VVV star count map. By scaling the total luminosity function (LF) obtained from all VVV fields to the LF from Zoccali et al.(2003), we obtain the first fully empirical estimate of the mass in stars and in remnants of the Galactic bulge. Within $\left(|b|<9.5^{\circ},|l|<10^{\circ}\right)$, the Milky Way bulge stellar mass is $2.0 \pm 0.3 \times 10^{10} M_{\odot}$.
\end{abstract}

Key words. Galaxy: structure - Galaxy: bulge

\section{Introduction}

Core helium-burning red clump (RC) stars are useful distance indicators, because their magnitude changes slowly and smoothly with age and metallicity, and is predicted very accurately by stellar evolution models (e.g., Salaris \& Girardi 2002). The RC is also a bright and distinct feature in the colour-magnitude diagram (CMD) of intermediate-to-old stellar populations, hence easy to identify. This makes it a powerful tracer of the Milky Way (MW) bulge structure. In particular, RC stars have been extensively used to trace the bar position, orientation and scale parameters (see the review of Gonzalez \& Gadotti 2015, for a complete list of references), as well as to uncover the presence of a peanut (X-shape) in the outer bulge (McWilliam \& Zoccali 2010; Nataf et al. 2010; Saito et al. 2011).

The infrared photometry from the VISTA Variables in the Vía Láctea (VVV) ESO public survey (Minniti et al. 2010) has been used by Wegg \& Gerhard (2013) to constrain the first $3 \mathrm{D}$ density model of the bulge. Their model was rather poorly constrained close to the plane because the DR1 catalogues (Saito et al. 2012) used in that study are based on aperture photometry, which suffers strongly from incompleteness because of the high level of crowding in the region $|b| \leq 0.5^{\circ}$.

\footnotetext{
* Based on observations taken within the ESO/VISTA Public Survey VVV under the programme ID 179.B-2002 (PI: Minniti).
}

Here we present the first stellar density map that reaches all the way to the centre of the MW bulge, using RC star counts from new VVV catalogs that are based on PSF-fitting photometry. We directly compare the stellar density with kinematics, and derive the first fully empirical estimate of the stellar mass of the bulge.

\section{Observation and data reduction}

Our data are part of the VVV survey that covers $\sim 180$ sq. deg of MW bulge between $|l| \leq 10^{\circ}$ and $|b| \leq 4.5^{\circ}$ contiguously. A detailed description of the global survey, and of the multiband observations completed within the first two years (used in this study), can be found in Minniti et al. (2010) and Saito et al. (2012), respectively.

Unlike most previous studies that are based on DR1 ${ }^{1}$ VVV data, we performed PSF-fitting photometry with the DoPHOT code (Schechter et al. 1993; Alonso-García et al. 2012) on each of the 16 chips in the images, both in $J$ and $K_{\mathrm{s}}$, and for this study we retrieved the stacked pawprint images from the CASU pipeline ${ }^{2}$, following Alonso-García et al. (2015).

\footnotetext{
1 Catalogs obtained from aperture photometry on individual tiles.

2 http://casu.ast.cam.ac.uk/
} 


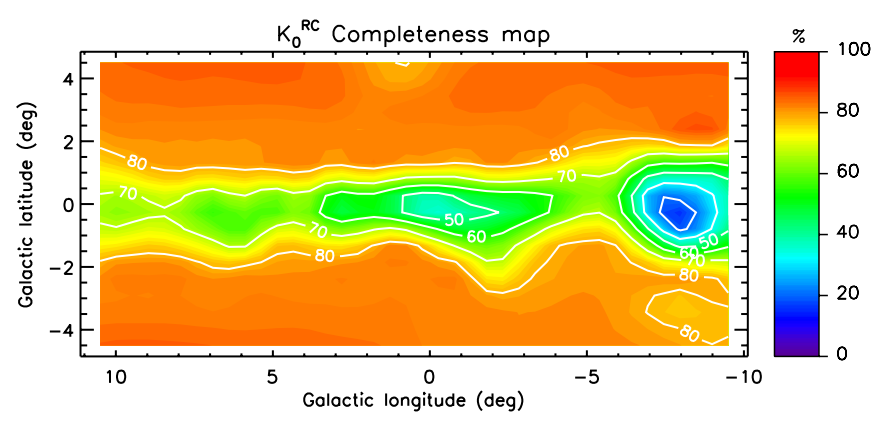

Fig. 1. Completeness map of the RC peak magnitude $\left(K_{0}^{\mathrm{RC}}\right)$ across the bulge.

The photometric catalogues of the individual chips were calibrated and astrometrized to the VISTA system. The six pawprint catalogs that cover a VISTA tile were combined into tile catalogs and the magnitudes of stars, which were detected multiple times, weight-averaged.

The photometric completeness was estimated via artificial star experiments. For each tile, stars were randomly added to chip\#12 only, assuming that the stellar density in this chip was representative of the whole tile. A more detailed description of the PSF photometry and completeness experiments for the whole VVV survey area is the subject of a forthcoming paper (Alonso-Garcia et al., in prep.). DoPHOT allowed us to obtain photometry with a typical error $<0.05$ down to $J \sim 18$ and $K_{\mathrm{s}} \sim 17$ in most of the bulge area. As expected, in the faint $\left(K_{\mathrm{s}}>17\right)$ magnitude regime, the completeness of the catalogs can vary significantly across the bulge area, being less complete for tiles closer to the plane, where both crowding and extinction are more severe. However, in the magnitude range of interest for the present work, i.e., $K_{0} \leq 14$, the derived catalogs are more than $70 \%$ complete, with the exception of a few fields (see Fig. 1).

\section{Analysis and results}

We aim to investigate the 3D structure and symmetry of the inner bulge in detail, by tracing the stellar density from RC star counts. To build an RC-based stellar density map, we needed to address two main issues that relate to the observing strategy that was adopted by VVV when surveying the bulge area. The first is that there is a non-negligible overlap $(\sim 10 \%)$ between adjacent tiles. We therefore cross-correlated adjacent tiles to eliminate double detections. The second issue is that the centers of the tiles are not symmetric with respect to the plane $\left(b=0^{\circ}\right)$, nor to the bulge projected minor axis $\left(l=0^{\circ}\right)$. Hence we resampled the bulge area analysed here with a new uniform grid of tiles, hereafter called fields, each with a $1^{\circ} \times 1^{\circ}$ area that is centred at integer values of both latitude and longitude between $-9.5^{\circ} \leq l \leq 10.5^{\circ}$ and $|b| \leq 4.5^{\circ}$. This allows us to directly and consistently compare fields that are located symmetrically around the bulge projected axes without the need for interpolations on stellar density or completeness.

The catalogs were corrected for extinction by applying the reddening map of Gonzalez et al. (2011b) and adopting the Nishiyama et al. (2005) extinction law. From the de-reddened catalog of each field, stars lying on the red giant branch (RGB) were selected using a variable color cut aimed at excluding stars in the bright $\left(K_{0} \leq 15\right)$ main sequence of the foreground disk, with a typical color $(J-K)_{0} \sim 0.3$. We then used RGB stars to derive the $K_{0}$ luminosity function (LF), corrected this for incompleteness, and measured the RC mean magnitude $\left(K_{0}^{\mathrm{RC}}\right)$ by following the prescription of Stanek et al. (1997). As such, the

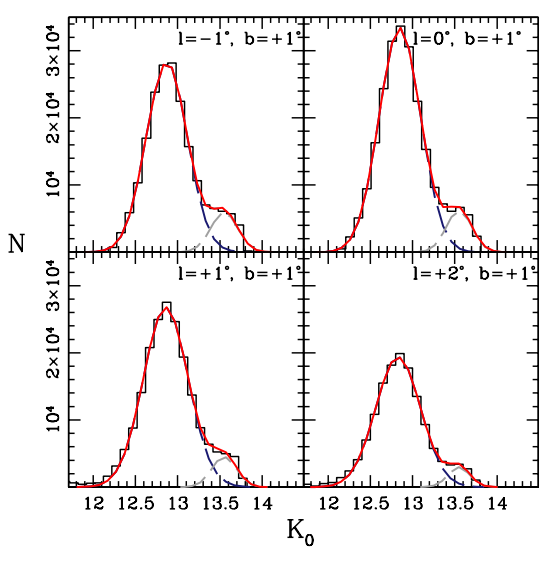

Fig. 2. $K_{0}$ LF (black histogram) for representative fields along the Galactic plane, after subtracting the contribution from the RGB stars. The red solid line shows the best fit to each LF, while the dashed lines identify the individual Gaussian fits to the RC (blue) and to the additional fainter peak (gray).

RGB LF was fitted with a second-order polynomial, together with a Gaussian to account for the peak of RC stars. In addition, as done by Nataf et al. (2011), Gonzalez et al. (2011b) and Wegg \& Gerhard (2013), a second Gaussian was included in the fit to account for the presence of a second peak, which is less populated and fainter than the RC (Fig. 2). The position and relative intensity of the second peak is broadly consistent with the expected position of the RGB bump (Sweigart et al. 1990; Wegg \& Gerhard 2013). However, as Gonzalez et al. (2011b), we noticed that the magnitude difference between the bulge $\mathrm{RC}$ and this faint peak is not constant close to the Galactic plane, as one would expect if it were a stellar evolutionary feature. The present data allowed us to map both the magnitude and the density of this second peak with respect to the bulge $\mathrm{RC}$, and will be the subject of a forthcoming paper.

\subsection{The $R C$ density map}

By using the total number of RC stars detected in all the fields, normalized to the their maximum value across the whole area, we derive the stellar density map shown in Fig. 3. As expected, the map clearly shows a boxy/peanut bulge, with an increase of star counts towards the center. The coloured circles on top of the grayscale map show the GIBS fields (Zoccali et al. 2014), where radial velocities were measured for 200-400 individual RC stars per field. The symbols are colour-coded according to the radial velocity dispersion measured in each of them. It is clear that the velocity dispersion follows the density of RC stars, and hence, the mass density. Therefore, the present map confirms the presence of a high stellar density peak in the central $\sim 150 \mathrm{pc}$, although the peak is more axisymmetric than the one extrapolated using the GIBS fields (see their Fig. 11).

In general, the vertical extent of the isodensity contours is larger at positive longitude, as expected for a bar that has its close side at positive longitude. This effect was previously noted by Alard (2001) using 2MASS photometry, although limited to $|b| \geq$ $2^{\circ}$. The high quality of the VVV PSF-fitting photometry allows us to map the stellar density of the innermost bulge regions $(|b| \leq$ $1.5^{\circ}$ ) for the first time. The maximum density of stars is found in the region $|l| \leq 1^{\circ}$ and $|b| \leq 0.5^{\circ}$, and, as shown by the 0.8 and 0.7 isodensity curves, appears slightly asymmetric with respect to the bulge's minor axis. The central asymmetry becomes more noticeable as we progressively move outwards. In fact, as shown in Fig. 4 , in the region between $|l| \leq 5^{\circ},|b| \leq 2^{\circ}$ we find that 


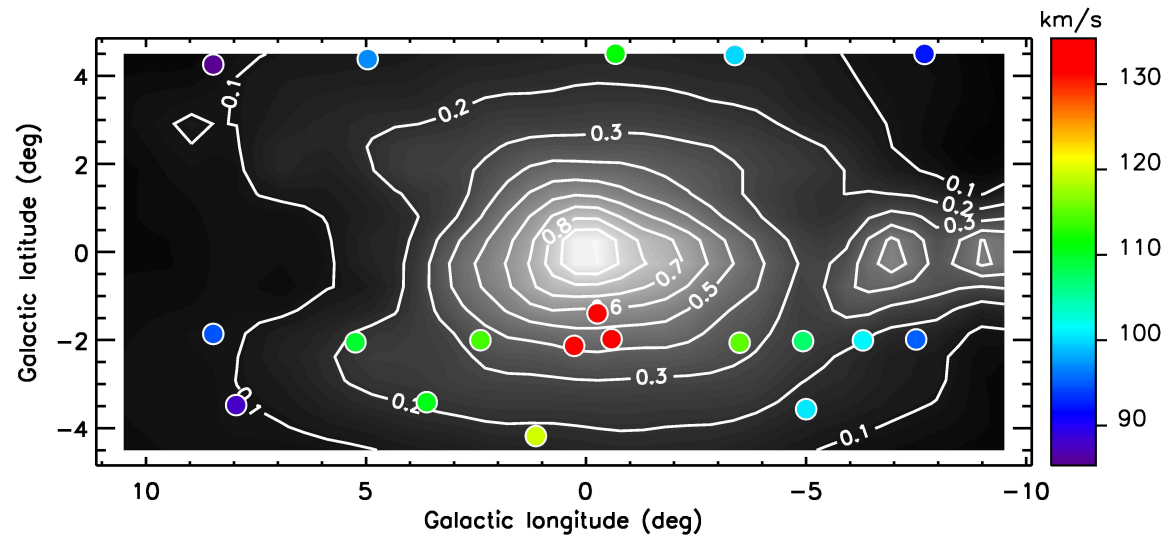

Fig. 3. Density map in the longitude-latitude plane based on RC star counts. Star counts have been normalized to the maximum (Max). Solid contours are isodensity curves, linearly spaced by $0.1 \times \mathrm{Max} \mathrm{deg}^{-2}$. The fields observed by GIBS are shown as circles, whose colors follow the measured velocity dispersion $(\sigma$ in $\mathrm{km} \mathrm{s}^{-1}$ ) in that given field.

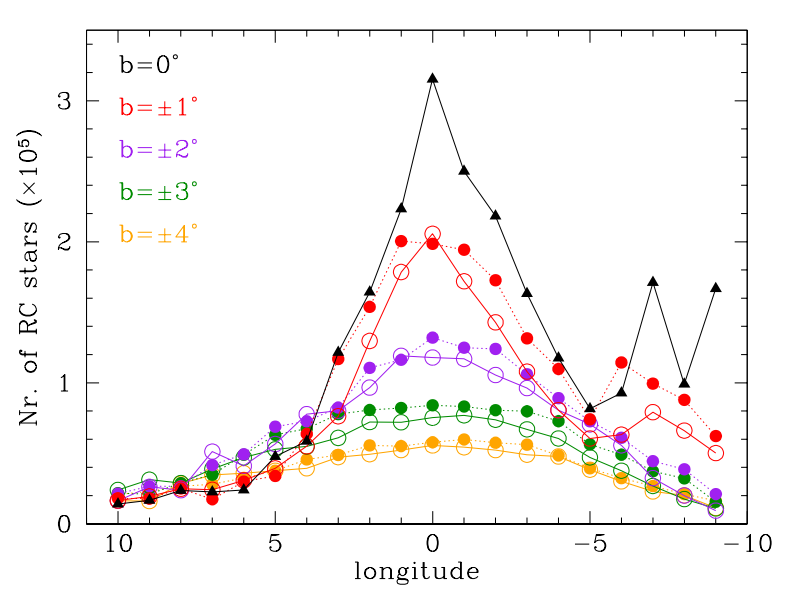

Fig. 4. Stellar density profile across the longitude, at fixed latitudes. Filled symbols with dotted lines show star counts at negative latitudes, while empty symbols and solid lines refers to positive latitudes.

the counts are systematically higher at $b<0$. The additional overdensity along the Galactic plane at $l \sim-7^{\circ}$ is also present in the GLIMPSE $4.5 \mu \mathrm{m}$ map (Churchwell et al. 2009). Finally, as discussed before, the absolute density of the central field and the field at $\left(-7^{\circ}, 0^{\circ}\right)$ should be taken as a lower limit because these are the only two fields for which the completeness corrections at $K_{0}^{\mathrm{RC}}$ are larger than $50 \%$ (Fig. 1 ).

\subsection{A fully empirical estimate of the bulge stellar mass}

In what follows we present a fully empirical estimate of the stellar mass of the bulge within the VVV area. We use the empirical initial mass function (IMF) measured by Zoccali et al. (2000) (recently confirmed by Calamida et al. 2015) in a HST/NICMOS field at $\left(0,-6^{\circ}\right)$, integrated to yield the mass contained in that field, and scale it to the whole bulge using the ratio of RC stars. The field of view (FoV) of NICMOS is too small to sample evolved stars, but a complete LF in a larger area $\left(\sim 8^{\prime} \times 8^{\prime}\right.$; from NTT/SOFI), including the NICMOS field, is presented in Zoccali et al. (2003). The scaling factor between NICMOS and SOFI $(\times 609)$ is simply the ratio of the FoV areas, given that they sample the same bulge region. From this scaling factor, we can derive the mass contained in the SOFI field. Finally, to obtain the total stellar mass in the VVV area, we measure the scaling factor between the number of RGB+RC stars in the whole VVV area and in the SOFI field.
In practice, the LF for the VVV catalogues was restricted to the RGB at the RC level $\left(K_{0}^{\mathrm{RC}} \pm 1.5 \mathrm{mag}\right)$ where the completeness is high (and thus the correction small) across the whole bulge area. Because the RC magnitude varies across the fields, as a result of the bar orientation, we measured the RC position in each field individually and counted stars within \pm 1.5 mag of the peak. This procedure enabled us to obtain a spatial mass profile in $1^{\circ}$ bins (Fig. 5). In the small region, where the RC is split because of the X-shape of the bulge $\left(|l|<4^{\circ}\right.$, for $\left.b<-5^{\circ}\right)$, we use the mean value of the two RCs. This is accurate enough for our purpose, which is just to find a normalization factor between the RGB in the SOFI and in the whole VVV area. Furthermore, we can assume symmetry around the Galactic plane to extend our mass estimate to the $\sim$ squared region, - hereafter $\mathrm{VVV}_{\square}-$ $|l|<10^{\circ}$ and $|b|<9.5^{\circ}(\sim 2.8 \times 2.7 \mathrm{kpc}$, assuming a distance to the Galactic center of $8 \mathrm{kpc})$. In other words, the number of $\mathrm{RGB}+\mathrm{RC}$ stars for $b>4.5^{\circ}$, outside the VVV area, is assumed to be equal to that at $b<-4.5^{\circ}$. Finally, since the LF from the SOFI field was decontaminated of disk stars (amounting to $18 \%$ within \pm 1.5 mag around the RC), the number of RGB+RC stars in $\mathrm{VVV}_{\square}$ was also reduced by this quantity. The ratio between the total number of $\mathrm{RGB}+\mathrm{RC}$ stars within $\pm 1.5 \mathrm{mag}$ from the $\mathrm{RC}$ in $\mathrm{VVV}_{\square} \mathrm{Vs}$. SOFI is 58624.

In Zoccali et al. (2000) the IMF was integrated using the observational data for main-sequence stars (from the turnoff down to $0.15 M_{\odot}$ ) and different assumptions for the IMF above $1 M_{\odot}$ and below $0.15 M_{\odot}$. From recent determinations of the IMF of massive stars (see Bastian et al. 2010, for a review), we assume a Salpeter IMF $(\alpha=-2.35)$ above $1 M_{\odot}$ and a power law with a slope of $\alpha=-0.3$ in the substellar regime. With the same initial-to-final-mass relation as in Zoccali et al. (2000), we found $570 M_{\odot}$ in the NICMOS field, hence $570 \times 609 \times 58624=$ $2.0 \times 10^{10} M_{\odot}$ in $\mathrm{VVV}_{\square}$. This is the mass in stars and remnants of the Galactic bulge in the region $|b|<9.5^{\circ},|l|<10^{\circ}$. It assumes a uniform disk contamination (at the level of $18 \%$ close to the RC) across the whole bulge area. While this assumption is accurate for fields close to $\left(0,-6^{\circ}\right)$, it is likely an overestimate for regions closer to the Galactic plane, and an underestimate for outer regions $^{3}$. Currently, an accurate estimate of the disk-to-bulge fraction for the inner few kpc of the Galaxy is not yet available. However, by adopting a smoothly varying disk contamination from $20 \%$ at $b= \pm 9^{\circ}$ down to $5 \%$ at $b= \pm 2^{\circ}$, and then back to $20 \%$ at $b=0^{\circ}$ where the thin disk might become significant

3 The disk contamination in three fields at different latitudes was estimated in Zoccali et al. (2008) using the Besançon model. At $\left(0,-6^{\circ}\right)$ it is very close to $20 \%$, in Baade's Window it is significantly smaller, while at $\left(0,-12^{\circ}\right)$ is larger (cf., their Table 4$)$. 


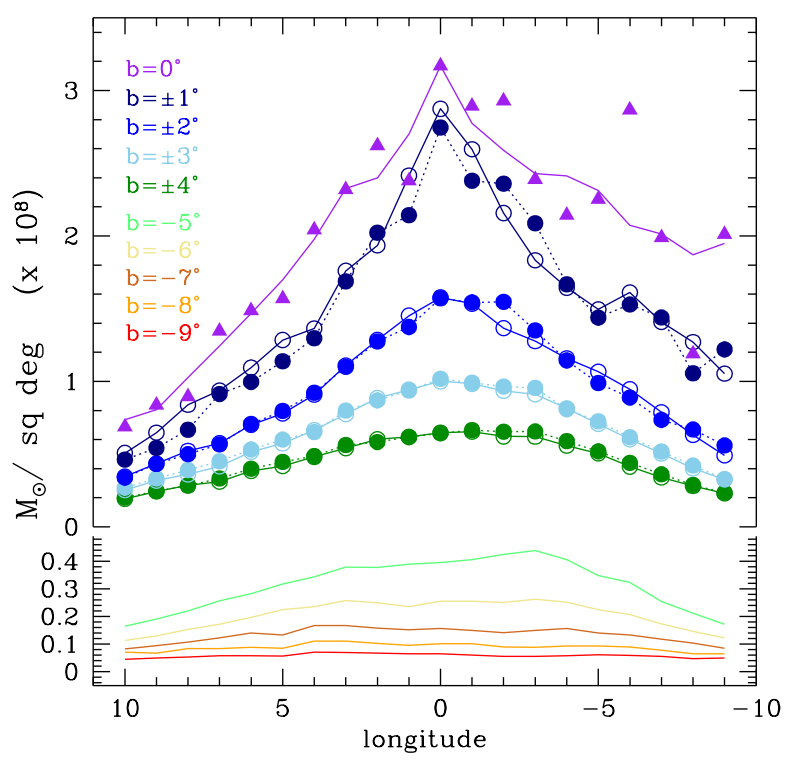

Fig. 5. Stellar mass profile across latitude in each field for fixed longitudes. Filled symbols with dotted lines represent $b<0$, empty symbols with solid lines to $b>0$ fields. The lower part of the plot has an expanded $y$-axis, for a better display of the high negative latitude fields. The number counts at $b=0^{\circ}$ are rather noisy, especially at negative longitudes, owing to the higher extinction, hence higher incompleteness in those fields. The purple line includes a mild smoothing from which the central point was excluded, to avoid flattening the peak.

again (e.g., Dékány et al. 2015), the total mass would only increase by $7 \%$. Other systematic error sources, such as the slope of the IMF above $1 M_{\odot}$ and below $0.08 M_{\odot}$, or the initial-to-final mass, are estimated as follows. A generous \pm 0.3 dex variation of the assumed IMF slope changes the total mass by less than $12 \%$. We further assume that small gradients on the bulge's dominant stellar populations do not change the number ratio between $\mathrm{RC}$ and main-sequence stars by more than a few percent. In summary, a conservative total uncertainty amounts to $15 \%$, which yields a total bulge mass within $V V V_{\square}$ of $2.0 \pm 0.3 \times 10^{10} M_{\odot}$, including disk contamination, extrapolation of the IMF, mass of the remnants, and stellar population gradients.

\section{Conclusions}

We have used the VVV $J$ and $K_{\mathrm{s}}$ single-epoch data to derive accurate PSF-fitting photometry in the bulge region between $-9.5^{\circ} \leq l \leq 10.5^{\circ}$ and $|b| \leq 4.5^{\circ}$. The photometric accuracy of the catalogs, which include modest incompleteness corrections (the result of crowding and extinction) allowed us to build the first density profile of the inner bulge towards the Galactic plane by means of RC star counts. We found a good agreement between the measured RC density distribution and the velocity dispersion map provided by the GIBS survey. In particular, the central high $\sigma$-peak at $|l| \leq 2^{\circ}$ and $|b| \leq 3^{\circ}$ in the derived velocity dispersion curve is well matched by a high stellar density peak in the central $\sim 150 \mathrm{pc}$. This tight link between the stellar density profile and the velocity dispersion map confirms that the flattening of the $K_{0}^{\mathrm{RC}}$ trend with longitude, for $|l| \leq 4^{\circ}$, (Nishiyama et al. 2005; Gonzalez et al. 2011a) is more likely the result of an inner compact and axisymmetric spheroid rather than the presence of a nuclear bar (Gerhard \& Martinez-Valpuesta 2012).

We further exploit the depth and high quality of the new VVV photometry that also extends to the innermost bulge regions, and derive the first purely empirical estimate of the bulge stellar mass by scaling the observed RC stellar density map with the observed bulge LF from Zoccali et al. (2000), Zoccali et al. (2003). We measure the mass in star and stellar remnants of the Galactic bulge as $2.0 \pm 0.3 \times 10^{10} M_{\odot}$. Previous bulge mass estimates, all model-dependent, span a considerable range from $6.1 \times 10^{9}$ (Robin et al. 2012) to $1.6 \times 10^{10} M_{\odot}($ Nesti \& Salucci 2013; Sofue 2013; Portail et al. 2015). A possible reason why our result, although compatible within the quoted errors, is larger than the mass estimated by dynamical models (e.g. Portail et al. 2015) is because our sampled volume is not limited along the line of sight. In addition, the kinematics used so far to constrain the models, do not extend to the innermost bulge regions $\left(|l| \leq 1^{\circ}\right.$ and $\left.b=-1^{\circ},-2^{\circ}\right)$, where a velocity dispersion peak was found (Zoccali et al. 2014).

Acknowledgements. The authors gratefully acknowledge the use of data from the ESO Public Survey program ID 179.B-2002, taken with the VISTA telescope, and data products from the Cambridge Astronomical Survey Unit. Support for M.Z. and D.M. is provided by the BASAL CATA Center for Astrophysics and Associated Technologies through grant PFB-06, and the Ministry for the Economy, Development, and Tourism's Programa Iniciativa Cientifica Milenio through grant IC120009, awarded to Millenium Institute of Astrophysics (MAS). D.M. and M.Z. acknowledge support from FONDECYT regular grant No. 1130196 and 1150345 , respectively. J.A.G. acknowledges support by the FIC-R Fund, allocated to Project 30321072. M.H. acknowledges financial support from the Chilean BASAL Centro de Excelencia en Astrofisica y Tecnologias Afines (CATA), grant PFB-06/2007.

\section{References}

\section{Alard, C. 2001, A\&A, 379, L44}

Alonso-García, J., Mateo, M., Sen, B., et al. 2012, AJ, 143, 70

Alonso-García, J., Dékány, I., Catelan, M., et al. 2015, AJ, 149, 99 Bastian, N., Covey, K. R., \& Meyer, M. R. 2010, ARA\&A, 48, 339 Calamida, A., Sahu, K. C., Casertano, S., et al. 2015, ApJ, 810, 8

Churchwell, E., Babler, B. L., Meade, M. R., et al. 2009, PASP, 121, 213

Dékány, I., Minniti, D., Majaess, D., et al. 2015, ApJ, 812, L29

Gerhard, O., \& Martinez-Valpuesta, I. 2012, ApJ, 744, L8

Gonzalez, O. A., \& Gadotti, D. A. 2015, in Galactic Bulges, eds. E. Laurikainen,

R. Peletier, D. Gadotti (Springer Publishing), in press [arXiv: 1503.07252] Gonzalez, O. A., Rejkuba, M., Minniti, D., et al. 2011a, A\&A, 534, L14

Gonzalez, O. A., Rejkuba, M., Zoccali, M., Valenti, E., \& Minniti, D. 2011b, A\&A, 534, A3

McWilliam, A., \& Zoccali, M. 2010, ApJ, 724, 1491

Minniti, D., Lucas, P. W., Emerson, J. P., et al. 2010, New Astron., 15, 433

Nataf, D. M., Udalski, A., Gould, A., Fouqué, P., \& Stanek, K. Z. 2010, ApJ, $721, \mathrm{~L} 28$

Nataf, D. M., Udalski, A., Gould, A., \& Pinsonneault, M. H. 2011, ApJ, 730, 118

Nesti, F., \& Salucci, P. 2013, J. Cosmol. Astropart. Phys., 7, 16

Nishiyama, S., Nagata, T., Baba, D., et al. 2005, ApJ, 621, L105

Portail, M., Wegg, C., Gerhard, O., \& Martinez-Valpuesta, I. 2015, MNRAS, 448, 713

Robin, A. C., Marshall, D. J., Schultheis, M., \& Reylé, C. 2012, A\&A, 538, A106

Saito, R. K., Zoccali, M., McWilliam, A., et al. 2011, AJ, 142, 76

Saito, R. K., Hempel, M., Minniti, D., et al. 2012, A\&A, 537, A107

Salaris, M., \& Girardi, L. 2002, MNRAS, 337, 332

Schechter, P. L., Mateo, M., \& Saha, A. 1993, PASP, 105, 1342

Sofue, Y. 2013, PASJ, 65, 118

Stanek, K. Z., Udalski, A., Szymański, M., et al. 1997, ApJ, 477, 163

Sweigart, A. V., Greggio, L., \& Renzini, A. 1990, ApJ, 364, 527

Wegg, C., \& Gerhard, O. 2013, MNRAS, 435, 1874

Zoccali, M., Cassisi, S., Frogel, J. A., et al. 2000, ApJ, 530, 418

Zoccali, M., Renzini, A., Ortolani, S., et al. 2003, A\&A, 399, 931

Zoccali, M., Hill, V., Lecureur, A., et al. 2008, A\&A, 486, 177

Zoccali, M., Gonzalez, O. A., Vasquez, S., et al. 2014, A\&A, 562, A66 